\title{
Tagungsbericht
}

Isabelle Dorsch*

\section{Trends der Informationswissenschaft: Suchmaschinen und Information Retrieval}

https://doi.org/10.1515/iwp-2019-2025

Am 26. April 2019 fand das erste Symposium Information Science Trends: Search Engines and Information Retrieval, ausgerichtet vom European Chapter der Association for Information Science and Technology (ASIS\&T) an der Hochschule für Angewandte Wissenschaften (HAW) statt. Dem Veranstaltungstitel entsprechend, konzentrierten sich die über 50 Teilnehmenden in spannenden Vorträgen und Diskussionen ganz auf den Themenbereich der Suchmaschinen und computergestützten Suche von Informationen. Die englischsprachige Tagesveranstaltung richtete sich dabei an alle Wissenschaftlerinnen und Wissenschaftler, Studierende und Fachkräfte aus der Wirtschaft. Interessierte, denen es nicht möglich war, vor Ort zu sein, konnten die Vorträge über einen Live-Stream verfolgen.

\section{Aktuelles aus dem Bereich der Suchmaschinen und computergestützten Suche von Informationen}

Nach einer kurzen Begrüßung durch die Moderatoren Dirk Lewandowski (HAW Hamburg), Aylin Ilhan und Isabelle Dorsch (Heinrich-Heine-Universität Düsseldorf) vom ASIS\&T European Chapter folgte der Keynote-Vortrag „Unsichtbare Suche im Alltag“ von Olof Sundin (Universität Lund, Schweden) (Abb. 1). Sundin thematisierte hierbei, wie der Alltag die computergestützte Suche und das Suchverhalten innerhalb der Gesellschaft beeinflusst. In der heutigen Zeit ist es für uns ganz normal mit einer kurzen Suche Informationen abzurufen, zum Beispiel darüber, wann der nächste Bus kommt oder was man zum Abendessen zubereiten kann. Dass dies in der Vergangenheit nicht immer so war und ein Wandel des Suchens statt-

*Kontaktperson: Isabelle Dorsch, Heinrich-Heine-Universität Düsseldorf, Abteilung für Informationswissenschaft, Universitätsstraße 1, 40225 Düsseldorf, E-Mail: Isabelle.Dorsch@hhu.de gefunden hat beziehungsweise immer noch stattfindet, zeigte Sundin mit Hilfe von Interviewzitaten aus seinen Forschungsprojekten. So ist es für eine der interviewten Person inzwischen reine Routine: „Früher war es noch etwas aufregend einen Computer zu nutzen, aber heute ist es so, als würde ich die Tageszeitung lesen, ich sitze einfach da und scrolle“. Ein weiteres Zitat beschreibt, dass nicht wirklich oder nur manchmal reflektiert wird, warum der erste Treffer der erste Treffer in der Liste ist und dass man Google „einfach vertraut“. Diese und viele weitere Beispiele in seinem Vortrag sind dabei ebenfalls basierend auf den Erkenntnissen aus Jutta Haider und Sundins neuem Buch „Invisible Search and Online Search Engines: The ubiquity of search in everyday life“ (Haider \& Sundin, 2019).

Wie kann man eine Suchmaschine und ihre Suche fair gestalten? Weiter ging es mit dieser Fragestellung, die Dirk Lewandowski in seinem Vortrag thematisierte. Nach einer kurzen Einführung zu Trefferlisten von Suchmaschinen und der visuellen Wahrnehmung von Suchergebnissen durch den Nutzer sprach Lewandowski über bestehende Arten von Verzerrungen von Trefferlisten, sogenannte Suchmaschinen-Biases. Ganz allgemein handelt es sich dabei um die Tendenz von Suchmaschinen, bestimmte Suchtreffer basierend auf ihren Algorithmen zu bevorzugen (Lewandowski, 2017). Wenngleich es empirische Fallstudien über die Verzerrung von Suchergebnissen z.B. hinsichtlich der Herkunft, des Geschlechts oder auch in Bezug auf Gesundheitsinformationen gibt, fehlen laut Lewandowski valide empirische Messungen solcher Suchmaschinenverzerrungen. Gefolgt von vielen weiteren Beispielen bleibt schlussendlich die Frage, was kann man tun, um solchen Verzerrungen entgegen zu wirken und die Suche fairer $\mathrm{zu}$ gestalten? Möglich wäre nach Lewandowski eine Regulation von Suchmaschinen wie Google, damit diese neutrale Suchergebnisse liefern und offensichtliche Verzerrungen sowie unlauteren Wettbewerb korrigieren. Des Weiteren ist es wichtig, die Informationskompetenz des Nutzers zu steigern und ihm mehr Auswahlmöglichkeiten und Transparenz bei der Nutzung einer Suchmaschine anzubieten. Die Entwicklung von weiteren oder alternativen Suchmaschinen (beispielsweise 

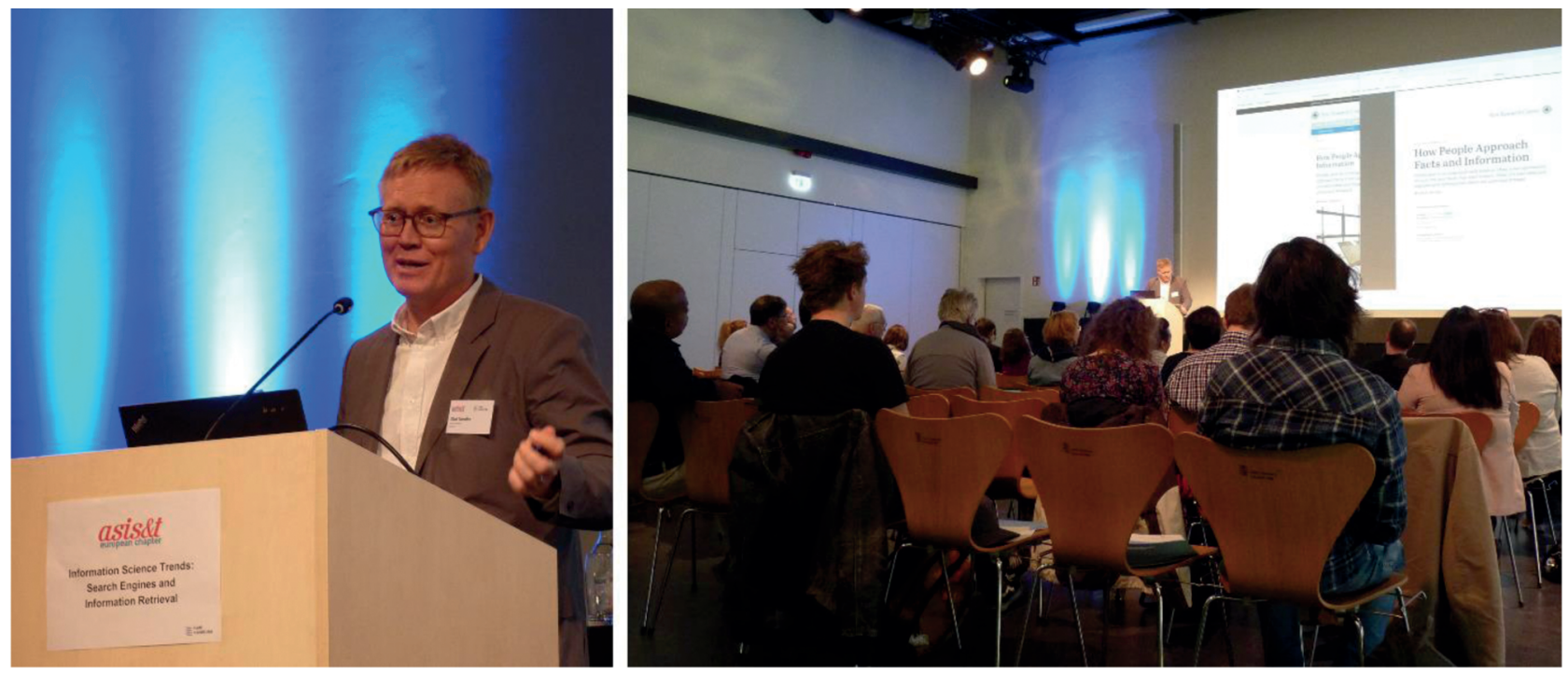

Abbildung 1: Keynote-Speaker Olof Sundin (Universität Lund, Schweden) über die unsichtbaren Suchen im Alltag. (Bild: Isabelle Dorsch)

nicht kommerzieller Herkunft) wäre ebenfalls eine Option, um das Suchen und Finden fairer zu gestalten.

Nach der Mittagspause gab es einen konkreten Einblick in die Praxis. Emmy Le (OTTO GmbH \& Co. KG Hamburg, Deutschland) erläuterte in ihrem Vortrag die Suchmaschine des Onlinekatalogs der Firma OTTO. Mit insgesamt über 2,9 Millionen Produkten aus den unterschiedlichsten Bereichen ist das 1949 gegründete Unternehmen einer der größten Onlinehändler auf dem deutschen Markt. Dabei ging Le nicht nur auf die Funktionalität der hauseigenen Suchmaschine ein, sondern thematisierte die damit verbundenen Herausforderungen im Onlinehandel, wie zum Beispiel die korrekte Zuordnung von Suchanfragen zu ihren entsprechenden Produkten. Ebenfalls sind die Evaluation und stetige Verbesserung des Onlinekatalogs ein wichtiger Aufgabenbereich für OTTO.

Nachfolgend boten die insgesamt vier Kurzpräsentationen eine Vielzahl an Einblicken in die aktuelle Forschung und Praxis. Den Anfang machte Astrid Mager (Institut für Technikfolgen Abschätzung, Wien) mit der Frage „Alternative Suchmaschinen als Antrieb für den sozialen Wandel?" - welche Motivationen, Wertesysteme und Visionen treiben die Entwicklung von europäischen Suchmaschinen und Projekten in diesem Bereich voran. Ingo Knuth (Hochschule für Medien, Kommunikation und Wirtschaft, Berlin) referierte, auch im Namen seiner Ko-Autorin Janina Masuhr, über „Entscheidungstreiber für die Nutzung von Suchmaschinen“ zur Rolle von Lock-in-Effekten. Knuth erklärte, dass bei Lock-in-Effekten Kunden abhängig von Marken, bestimmten Gewohnheiten oder Softwaresystemen sind. Die zentrale Forschungsfrage der beiden, ob die Auswahl der Suchmaschine im Zusammenhang mit Lock-in-Effekten wie die Wahrnehmung der Mar- ken und die Integration der Plattform steht, konnte Knuth in seinem Vortrag mit einem ,ja“ beantworten. „Einflüsse auf den Beurteilungsprozess von Relevanz in akademischen Suchsystemen“ beschäftigen Christiane Behnert (HAW Hamburg) im Rahmen ihrer Dissertation. Im Fokus stand die Frage, nach welchen Kriterien Nutzer von akademischen Suchsystemen die Relevanz eines Suchergebnisses beurteilen. Kawa Nazemi (Hochschule Darmstadt) präsentierte in seinem Vortrag „Visuelle Trendanalyse in digitalen Bibliotheken“ sein System für eine visuelle Darstellung von Publikationsdaten zur Trend-Analyse. Höhepunkt seines Vortrages und gleichzeitiger Abschluss der Kurzpräsentationen war die Live-Vorstellung des Systems, die in einer anschließenden Diskussionsrunde und Kaffeepause mündete.

Bevor es mit dem für diesen Tag letzten thematisch fachlichen Vortrag weiterging, stellte Aylin Ilhan allen Teilnehmenden die Organisation ASIS\&T und das darin verankerte European Chapter vor.

Was genau ist Data Science? Mit dieser Fragestellung leitete Tom Alby (Euler Hermes, Hamburg) seinen Vortrag über „Data Science in der Suchmaschinenentwicklung“ ein. Eine exakte offizielle Definition existiere laut Alby nicht, es lässt sich jedoch als eine Mischung aus Statistik, Machine Learning und Data Mining definieren. Dem Vortragstitel entsprechend, bezog sich Alby konkret auf Data Science im Bereich der Suche und Suchmaschinen. So erklärte der Data Scientist Alby, der schon für mehrere renommierte Suchmaschinenfirmen wie beispielsweise Google gearbeitet hat, sehr anschaulich, wie Machine Learning im Allgemeinen funktioniert und wie genau eine Support Vector Machine für das Ranking von Suchmaschinentreffern genutzt werden kann. 
Die Tagesveranstaltung bot den Teilnehmern durch interessante Vorträge eine spannende Diskussionsgrundlage, um sich über aktuelle Forschungsthemen im Bereich der Suchmaschinen und computergestützten Forschung auszutauschen. So spannend, dass dieser Austausch von manchen Teilnehmenden nach dem Event in kleiner Runde und mit leckerem indischem Essen fortgeführt wurde.

\section{Über die Event-Reihe „Information Science Trends"}

Das Symposium in Hamburg bildete den Start einer neuen Event-Serie „Information Science Trends“ des ASIS\&T European Chapters. Ziel ist es, Forschenden, Studierenden und Fachkräften aus der Wirtschaft sowie Unternehmen aus Europa eine Plattform für informationswissenschaftliche sowie verwandte Forschung $\mathrm{zu}$ einem spezifischen Thema zu geben. Das European Chapter möchte dabei unterstützend tätig sein und plant die Event-Reihe mit wechselnden Themen und Veranstaltungsorten fortzuführen. Interessierte sind herzlich dazu eingeladen, Ideen und Vorschläge für weitere Events dieser Reihe dem ASIS\&T European Chapter weiterzuleiten.

\section{Links}

Informationen über das Veranstaltungsprogramm, Links $\mathrm{zu}$ Videomitschnitten und zukünftige Events sind $\mathrm{zu}$ finden unter: http://www.asis.org/Chapters/europe/

Deskriptoren: Tagung, Wissenschaft, Informationssuche, Suchmaschine, Data Sience

\section{Literatur}

Haider, J. \& Sundin, O. (2019). Invisible Search and Online Search Engines: The Ubiquity of Search in Everyday Life. London \& New York: Routledge.

Lewandowski, D. (2017). Users' Understanding of Search Engine Advertisements. Journal of Information Science Theory and Practice, 5(4), 6-25.

\section{Isabelle Dorsch}

Heinrich-Heine-Universität Düsseldorf

Abteilung für Informationswissenschaft

Universitätsstraße 1

40225 Düsseldorf

Isabelle.Dorsch@hhu.de 